\title{
14 Analysis of Boundary-domain Integral and Integro-differential Equations for a Dirichlet Problem with a Variable Coefficient
}

\author{
Sergey E. Mikhailov
}

\subsection{Introduction}

The Dirichlet boundary value problem for the "Laplace" linear differential equation with variable coefficient is reduced to boundary-domain integral or integro-differential equations (BDIEs or BDIDEs) based on a specially constructed parametrix. The BDI(D)Es contain potential-type integral operators defined on the domain under consideration and acting on the unknown solution as well as integral operators defined on the boundary and acting on the trace and/or conormal derivative of the unknown solution or on an auxiliary function. Some of the considered BDIDEs are to be supplemented by the original boundary conditions, thus constituting boundary-domain integro-differential problems (BDIDPs). Solvability, solution uniqueness, and equivalence of the BDIEs/BDIDEs/BDIDPs to the original boundary value problem (BVP) are investigated in appropriate Sobolev spaces.

Reduction of boundary-value problems with arbitrarily variable coefficients to boundary integral equations is usually not effective for numerical implementations, since the fundamental solution necessary for such reduction is generally not available in an analytical form (except some special dependence of the coefficients on coordinates, see e.g. [1]). Using a parametrix (Levi function) as a substitute of a fundamental solution, it is possible however to reduce such a BVP to a BDIE integral equation (see, e.g., [2], [3], [4, Sect. 18], [5], and [6], where the Dirichlet, Neumann, and Robin problems for some partial differential equations (PDEs) were reduced to indirect BDIEs).

In [7], [8], and [10], the 3D mixed (Dirichlet-Neumann) BVP for the variable-coefficient "Laplace" equation was considered. Such equations appear, e.g., in electrostatics, stationary heat transfer and other diffusion problems for inhomogeneous media. The BVP has been reduced to either segregated or united direct BDI(D)Es or BDIDPs. Some of the BDI(D)Es/BDIDPs are associated with the BDIDE and BDIE formulated in [9]. Although several of the integral and integro-differential formulations 
for the mixed problem in [7], [8], and [10] look like equations of the second kind, the spaces for the out-of-integral terms are different from the spaces for the right-hand sides of the equations, thus the equations are of "almost" second kind.

Analysis of the four different (systems of) BDI(D)Es/BDIDP, to which the Dirichlet problem for the same PDE is reduced in the present paper, requires special consideration. Equivalence of the considered BDI(D)Es/ BDIDP to the original BVP is proved along with their solvability, solution uniqueness, and the operator invertibility in corresponding SobolevSlobodetski spaces. In particular, it is shown that the Dirichlet problem can be reduced to a genuine second-kind integral or integro-differential equation.

\subsection{Formulation of the Boundary Value Problem}

Let $\Omega$ be a bounded open three-dimensional region of $\mathbb{R}^{3}$. For simplicity, we assume that the boundary $S:=\partial \Omega$ is a simply connected, closed, infinitely smooth surface. Let $a \in C^{\infty}(\bar{\Omega}), a(x)>0$ for $x \in \bar{\Omega}$. Let also $\partial_{x_{j}}:=\partial / \partial x_{j}$ $(j=1,2,3), \partial_{x}=\left(\partial_{x_{1}}, \partial_{x_{2}}, \partial_{x_{3}}\right)$.

We consider the scalar elliptic differential equation

$$
\begin{aligned}
L u(x) & :=L\left(x, \partial_{x}\right) u(x) \\
& :=\sum_{i=1}^{3} \frac{\partial}{\partial x_{i}}\left(a(x) \frac{\partial u(x)}{\partial x_{i}}\right)=f(x), \quad x \in \Omega,
\end{aligned}
$$

where $u$ is an unknown function and $f$ is a given function in $\Omega$.

In what follows, $H^{s}(\Omega)=H_{2}^{s}(\Omega)$ and $H^{s}(S)=H_{2}^{s}(S)$ are the Bessel potential spaces, where $s \in \mathbb{R}$ is an arbitrary real number (see, e.g., [11] and [12]). We recall that $H^{s}$ coincide with the Sobolev-Slobodetski spaces $W_{2}^{s}$ for any nonnegative or integer $s$.

For a linear operator $L_{*}$, we introduce the subspace of $H^{1}(\Omega)$ [13]

$$
H^{1,0}\left(\Omega ; L_{*}\right):=\left\{g: g \in H^{1}(\Omega), L_{*} g \in L_{2}(\Omega)\right\},
$$

endowed with the norm

$$
\|g\|_{H^{1,0}\left(\Omega ; L_{*}\right)}:=\|g\|_{H^{1}(\Omega)}+\left\|L_{*} g\right\|_{L_{2}(\Omega)} .
$$

In this paper, we will particularly use the space $H^{1,0}\left(\Omega ; L_{*}\right)$ for $L_{*}$ being either the operator $L$ from (14.1) or the Laplace operator $\Delta$. Since

$$
L u-\Delta u=\sum_{i=1}^{3} \frac{\partial a}{\partial x_{i}} \frac{\partial u}{\partial x_{i}} \in L_{2}(\Omega)
$$

for $u \in H^{1}(\Omega)$, we have $H^{1,0}(\Omega ; L)=H^{1,0}(\Omega ; \Delta)$.

From the trace theorem (see, e.g., [11] and [13]-[15]) for $u \in H^{1}(\Omega)$, it follows that $u^{+}:=\tau_{S}^{+} u \in H^{1 / 2}(S)$, where $\tau_{S}^{+}$is the trace operator on $S$ from $\Omega$. 
For $u \in H^{2}(\Omega)$ we can denote by $T^{+}$the corresponding conormal differentiation operator on $S$ in the sense of traces,

$$
\begin{aligned}
T^{+}\left(x, n^{+}(x), \partial_{x}\right) u(x): & =\sum_{i=1}^{3} a(x) n_{i}^{+}(x)\left(\frac{\partial u(x)}{\partial x_{i}}\right)^{+} \\
& =a(x)\left(\frac{\partial u(x)}{\partial n^{+}(x)}\right)^{+}
\end{aligned}
$$

where $n^{+}(x)$ is the exterior (to $\Omega$ ) unit normal vectors at the point $x \in S$.

Let $u \in H^{1,0}(\Omega ; \Delta)$. We can correctly define the generalized conormal derivative $T^{+} u \in H^{-1 / 2}(S)$ with the help of Green's formula (see, for example, [13] and [15], Lemma 4.3),

$$
\begin{aligned}
\left\langle T^{+} u, v^{+}\right\rangle_{S}:= & \int_{\Omega} v(x) L u(x) d x \\
& +\int_{\Omega} \sum_{i=1}^{3} a(x) \frac{\partial u(x)}{\partial x_{i}} \frac{\partial v(x)}{\partial x_{i}} d x \quad \forall v \in H^{1}(\Omega),
\end{aligned}
$$

where $\langle\cdot, \cdot\rangle_{S}$ denotes the duality brackets between the spaces $H^{-1 / 2}(S)$ and $H^{1 / 2}(S)$, extending the usual $L_{2}$ scalar product.

We will investigate the following Dirichlet boundary value problem.

Find a function $u \in H^{1}(\Omega)$ satisfying the conditions

$$
\begin{array}{cl}
L u=f & \text { in } \Omega, \\
u^{+}=\varphi_{0} & \text { on } S,
\end{array}
$$

where $\varphi_{0} \in H^{1 / 2}(S)$ and $f \in L_{2}(\Omega)$.

Equation (14.3) is understood in the distributional sense and condition (14.4) in the trace sense.

We have the following uniqueness theorem.

Theorem 1. BVP (14.3)-(14.4) with $\varphi_{0} \in H^{1 / 2}(S)$ and $f \in L_{2}(\Omega)$ has at most one solution in $H^{1}(\Omega)$.

Proof. The assertion follows immediately from Green's formula (14.2) with $v=u$ as a solution of the homogeneous Dirichlet problem, i.e., with $f=0$ and $\varphi_{0}=0$.

\subsection{Parametrix and Potential-type Operators}

We say that a function $P(x, y)$ of two variables $x, y \in \Omega$ is a parametrix (the Levi function) for the operator $L\left(x, \partial_{x}\right)$ in $\mathbb{R}^{3}$ if (see, e.g., [2]-[6] and [9])

$$
L\left(x, \partial_{x}\right) P(x, y)=\delta(x-y)+R(x, y),
$$


where $\delta(\cdot)$ is the Dirac distribution and $R(x, y)$ possesses a weak (integrable) singularity at $x=y$, i.e.,

$$
R(x, y)=\mathcal{O}\left(|x-y|^{-\kappa}\right) \quad \text { with } \kappa<3 .
$$

It is easy to see that for the operator $L\left(x, \partial_{x}\right)$ given by the right-hand side in (14.1), the function

$$
P(x, y)=\frac{-1}{4 \pi a(y)|x-y|}, \quad x, y \in \mathbb{R}^{3},
$$

is a parametrix; the corresponding remainder function is

$$
R(x, y)=\sum_{i=1}^{3} \frac{x_{i}-y_{i}}{4 \pi a(y)|x-y|^{3}} \frac{\partial a(x)}{\partial x_{i}}, \quad x, y \in \mathbb{R}^{3},
$$

and satisfies estimate (14.6) with $\kappa=2$, due to the smoothness of the function $a(x)$.

Evidently, the parametrix $P(x, y)$ given by (14.7) is a fundamental solution to the operator $L\left(y, \partial_{x}\right):=a(y) \Delta\left(\partial_{x}\right)$ with "frozen" coefficient $a(x)=a(y)$, i.e.,

$$
L\left(y, \partial_{x}\right) P(x, y)=\delta(x-y) .
$$

Note that remainder (14.8) is not smooth enough for the parametrix (14.7) and the corresponding potential operators to be treated as in [15].

For some scalar function $g$, let

$$
\begin{gathered}
V g(y):=-\int_{S} P(x, y) g(x) d S_{x}, \quad y \notin S, \\
W g(y):=-\int_{S}\left[T\left(x, n(x), \partial_{x}\right) P(x, y)\right] g(x) d S_{x}, \quad y \notin S,
\end{gathered}
$$

be the single and the double layer surface potential operators, where the integrals are understood in the distributional sense if $g$ is not integrable.

The corresponding boundary integral (pseudodifferential) operators of direct surface values of the simple layer potential $\mathcal{V}$ and of the double layer potential $\mathcal{W}$, and the conormal derivatives of the simple layer potential $\mathcal{W}^{\prime}$ and of the double layer potential $\mathcal{L}^{+}$are

$$
\begin{gathered}
\mathcal{V} g(y):=-\int_{S} P(x, y) g(x) d S_{x}, \\
\mathcal{W} g(y):=-\int_{S}\left[T\left(x, n(x), \partial_{x}\right) P(x, y)\right] g(x) d S_{x},
\end{gathered}
$$




$$
\begin{gathered}
\mathcal{W}^{\prime} g(y):=-\int_{S}\left[T\left(y, n(y), \partial_{y}\right) P(x, y)\right] g(x) d S_{x}, \\
\mathcal{L}^{+} g(y):=\left[T\left(y, n(y), \partial_{y}\right) W g(y)\right]^{+},
\end{gathered}
$$

where $y \in S$.

The parametrix-based volume potential operator and the remainder potential operator, corresponding to parametrix (14.7) and to remainder (14.8) are

$$
\begin{aligned}
& \mathcal{P} g(y):=\int_{\Omega} P(x, y) g(x) d x, \\
& \mathcal{R} g(y):=\int_{\Omega} R(x, y) g(x) d x .
\end{aligned}
$$

For $g_{1} \in H^{-1 / 2}(S)$ and $g_{2} \in H^{1 / 2}(S)$, there hold the jump relations on $S$

$$
\begin{gathered}
{\left[V g_{1}(y)\right]^{+}=\mathcal{V} g_{1}(y)} \\
{\left[W g_{2}(y)\right]^{+}=-\frac{1}{2} g_{2}(y)+\mathcal{W} g_{2}(y),} \\
{\left[T\left(y, n(y), \partial_{y}\right) V g_{1}(y)\right]^{+}=\frac{1}{2} g_{1}(y)+\mathcal{W}^{\prime} g_{1}(y),}
\end{gathered}
$$

where $y \in S$.

The jump relations as well as mapping properties of potentials and operators (14.9)-(14.16) are well known for the case $a=$ const. They were extended to the case of variable coefficient $a(x)$ in [7] and [8].

\subsection{Green Identities and Integral Relations}

Let $u \in H^{1,0}(\Omega ; \Delta), v \in H^{1,0}(\Omega ; \Delta)$ be some real functions. Then, subtracting (14.2) from its counterpart with exchanged roles of $u$ and $v$, we obtain the so-called second Green identity for the operator $L\left(x, \partial_{x}\right)$,

$$
\int_{\Omega}\left[v L\left(x, \partial_{x}\right) u-u L\left(x, \partial_{x}\right) v\right] d x=\left\langle T^{+} u, v^{+}\right\rangle_{S}-\left\langle u^{+}, T^{+} v\right\rangle_{S}
$$

For $u \in H^{1,0}(\Omega ; \Delta)$ and $v(x)=P(x, y)$, where the parametrix $P(x, y)$ is given by (14.7), we obtain from (14.17), (14.5) by the standard limiting procedures (cf. [4]) the third Green identity,

$$
u(y)+\mathcal{R} u(y)-V T^{+} u(y)+W u^{+}(y)=\mathcal{P} L u(y), \quad y \in \Omega .
$$

If $u \in H^{1,0}(\Omega ; \Delta)$ is a solution of equation (14.1), then (14.18) gives

$$
G u:=u+\mathcal{R} u-V T^{+} u+W u^{+}=\mathcal{P} f \quad \text { in } \Omega,
$$




$$
\begin{gathered}
\mathcal{G} u:=\frac{1}{2} u^{+}+[\mathcal{R} u]^{+}-\mathcal{V} T^{+} u+\mathcal{W} u^{+}=[\mathcal{P} f]^{+} \quad \text { on } S, \\
\mathcal{T} u:=\frac{1}{2} T^{+} u+T^{+} \mathcal{R} u-\mathcal{W}^{\prime} T^{+} u+\mathcal{L}^{+} u^{+}=T^{+} \mathcal{P} f \quad \text { on } S
\end{gathered}
$$

For some functions $f, \Psi, \Phi$, let us consider a more general "indirect" integral relation, associated with (14.19), namely,

$$
u(y)+\mathcal{R} u(y)-V \Psi(y)+W \Phi(y)=\mathcal{P} f(y), \quad y \in \Omega .
$$

Lemma 1. Let $\Psi \in H^{-1 / 2}(S), \Phi \in H^{1 / 2}(S)$, and $f \in L_{2}(\Omega)$. Suppose a function $u \in H^{1}(\Omega)$ satisfies (14.22). Then $u \in H^{1,0}(\Omega ; \Delta)$, it is a solution of PDE (14.3) in $\Omega$, and

$$
V\left(\Psi-T^{+} u\right)(y)-W\left(\Phi-u^{+}\right)(y)=0, \quad y \in \Omega .
$$

Proof. First of all, equation (14.22) and mapping properties of the operators $\mathcal{R}, \mathcal{P}, V$ and $W$ imply $u \in H^{1,0}(\Omega ; \Delta)$. The rest of the lemma claims follow from its counterpart proved in [7], Lemma 4.1. $4.2)$.

The following statement is well known (see, for example, [7], Lemma

Lemma 2. (i) Let $\Psi^{*} \in H^{-1 / 2}(S)$. If $V \Psi^{*}(y)=0, y \in \Omega$, then $\Psi^{*}=0$.

(ii) Let $\Phi^{*} \in H^{1 / 2}(S)$. If $W \Phi^{*}(y)=0, y \in \Omega$, then $\Phi^{*}=0$.

Theorem 2. Let $f \in L_{2}(\Omega)$. A function $u \in H^{1,0}(\Omega ; \Delta)$ is a solution of $P D E$ (14.3) in $\Omega$ if and only if it is a solution of BDIDE (14.19).

Proof. If $u \in H^{1,0}(\Omega ; \Delta)$ solves PDE (14.3) in $\Omega$, then it satisfies (14.19). On the other hand, if $u \in H^{1,0}(\Omega ; \Delta)$ solves BDIDE (14.19), then Lemma 1 with $\Psi=T^{+} u$ and $\Phi=u^{+}$completes the proof.

\subsection{Segregated Boundary-domain Integral Equations}

Let us consider a segregated purely integral boundary-domain formulation for the Dirichlet problem, similar to the formulations introduced and analyzed in [7], [8], and [10] for the mixed problem with $u \in H^{1}(\Omega)$ and $u \in H^{1,0}(\Omega ; \Delta)$.

\subsubsection{Integral Equation System $(G \mathcal{G})$}

To reduce BVP (14.3)-(14.4) to a BDIE system in this section, we will use equation (14.19) in $\Omega$ and equation (14.20) on $S$, where the known function $\varphi_{0}$ is substituted for $u^{+}$and an auxiliary unknown function $\psi \in H^{-1 / 2}(S)$ for $T^{+} u$. Then we arrive at the system

$$
\begin{gathered}
u(y)+\mathcal{R} u(y)-V \psi(y)=F_{0}(y), \quad y \in \Omega, \\
\mathcal{R}^{+} u(y)-\mathcal{V} \psi(y)=F_{0}^{+}(y)-\varphi_{0}(y), \quad y \in S,
\end{gathered}
$$


where

$$
F_{0}(y):=\mathcal{P} f(y)-W \varphi_{0}(y), \quad y \in \Omega .
$$

Note that for $f \in L_{2}(\Omega)$ and $\varphi_{0} \in H^{1 / 2}(S)$, we have the inclusion $F_{0} \in H^{1,0}(\Omega ; \Delta)$ due to the mapping properties of the Newtonian (volume) and layer potentials.

Remark 1. $F_{0}=0$ if and only if $\left(f, \varphi_{0}\right)=0$. Indeed, the latter equality evidently implies the former. Inversely, let $F_{0}=0$. Keeping in mind equation (14.25), Lemma 1 with $F_{0}=0$ for $u$ implies $f=0$ and $W \varphi_{0}=0$ in $\Omega$. Lemma 2(ii) then gives $\varphi_{0}=0$ on $S$.

Let us prove that BVP (14.3)-(14.4) in $\Omega$ is equivalent to the system of BDIEs (14.23)-(14.24).

Theorem 3. Let $f \in L_{2}(\Omega)$ and $\varphi_{0} \in H^{1 / 2}(S)$.

(i) If some $u \in H^{1}(\Omega)$ solves $B V P(14.3)-(14.4)$ in $\Omega$, then the solution is unique and the couple $(u, \psi) \in H^{1}(\Omega) \times H^{-1 / 2}(S)$, where

$$
\psi=T^{+} u \quad \text { on } S,
$$

solves BDIE system (14.23)-(14.24);

(ii) If a couple $(u, \psi) \in H^{1}(\Omega) \times H^{-1 / 2}(S)$ solves BDIE system (14.23)(14.24), then the solution is unique, $u$ solves BVP (14.3)-(14.4), and $\psi$ satisfies (14.26).

Proof. Remark that if $u \in H^{1}(\Omega)$ is a solution of the BVP (14.3)-(14.4) with $f \in L_{2}(\Omega)$, then $u \in H^{1,0}(\Omega ; \Delta)$. On the other hand, if $\mathcal{U}=(u, \psi) \in$ $H^{1}(\Omega) \times H^{-1 / 2}(S)$ is a solution of system (14.23)-(14.24) with $f \in L_{2}(\Omega)$ and $\varphi_{0} \in H^{1 / 2}(S)$, then $u \in H^{1,0}(\Omega ; \Delta)$ due to mapping properties of operators $\mathcal{R}, V$, and $W$ (see [10] and [8]).

Let $u \in H^{1}(\Omega)$ be a solution to BVP (14.3)-(14.4). It is unique due to Theorem 1. Set $\psi$ by (14.26), evidently, $\psi \in H^{-1 / 2}(S)$. Then it immediately follows from relations (14.19)-(14.20) that the couple $(u, \psi)$ solves system (14.23)-(14.24), which completes the proof of item (i).

Let now a couple $(u, \psi) \in H^{1}(\Omega) \times H^{-1 / 2}(S)$ solve BDIE system (14.23)(14.24). Taking trace of equation (14.23) on $S$ and subtracting equation (14.24) from it, we obtain

$$
u^{+}(y)=\varphi_{0}(y), \quad y \in S,
$$

i.e., $u$ satisfies the Dirichlet condition (14.4).

Equation (14.23) and Lemma 1 with $\Psi=\psi$ and $\Phi=\varphi_{0}$ imply that $u$ is a solution of PDE (14.3) and

$$
V \Psi^{*}(y)-W \Phi^{*}(y)=0, \quad y \in \Omega,
$$

where $\Psi^{*}=\psi-T^{+} u$ and $\Phi^{*}=\varphi_{0}-u^{+}$. Due to equation (14.27), $\Phi^{*}=0$. Lemma 2(i) implies that $\Psi^{*}=0$, which completes the proof of conditions $(14.26)$. 
Uniqueness of the solution to BDIE system (14.23)-(14.24) follows from (14.26) along with Remark 1 and Theorem 1.

System (14.23)-(14.24) can be rewritten in the form

$$
\mathcal{A}^{G \mathcal{G}} \mathcal{U}=\mathcal{F}^{G \mathcal{G}}
$$

where $\mathcal{U}^{\top}:=(u, \psi) \in H^{1,0}(\Omega ; \Delta) \times H^{-1 / 2}(S)$,

$$
\mathcal{A}^{G \mathcal{G}}:=\left[\begin{array}{cc}
I-\mathcal{R} & -V \\
\mathcal{R}^{+} & -\mathcal{V}
\end{array}\right], \quad \mathcal{F}^{G \mathcal{G}}:=\left[\begin{array}{c}
F_{0} \\
F_{0}^{+}-\varphi_{0}
\end{array}\right] .
$$

Due to the mapping properties of operators $V, \mathcal{V}, W, \mathcal{W}, \mathcal{P}, \mathcal{R}$, and $\mathcal{R}^{+}$ (see [10] and [8]), we have $\mathcal{F}^{G \mathcal{G}} \in H^{1,0}(\Omega ; \Delta) \times H^{1 / 2}(S)$, and the operators

$$
\begin{aligned}
\mathcal{A}^{G \mathcal{G}} & : H^{1,0}(\Omega ; \Delta) \times H^{-1 / 2}(S) \rightarrow H^{1,0}(\Omega ; \Delta) \times H^{1 / 2}(S) \\
& : H^{1}(\Omega) \times H^{-1 / 2}(S) \rightarrow H^{1}(\Omega) \times H^{1 / 2}(S)
\end{aligned}
$$

are continuous. By Theorem 3 and the uniqueness Theorem 1, both operators (14.28) and (14.29) are injective.

Theorem 4. Operators (14.28) and (14.29) are continuous and invertible.

Proof. Let us consider the proof for the operator $\mathcal{A}^{G \mathcal{G}}$ given by (14.29) first. The continuity and injectivity are proved above. To prove the invertibility, let us consider the operator

$$
\mathcal{A}_{0}^{G \mathcal{G}}:=\left[\begin{array}{cc}
I & -V \\
0 & -\mathcal{V}
\end{array}\right] .
$$

As a result of compactness properties of the operators $\mathcal{R}$ and $\mathcal{R}^{+}$, the operator $\mathcal{A}_{0}^{G \mathcal{G}}$ is a compact perturbation of the operator $\mathcal{A}^{G \mathcal{G}}$.

The operator $\mathcal{A}_{0}^{G \mathcal{G}}$ is an upper triangular matrix operator with the scalar diagonal invertible operators

$$
\begin{gathered}
I: H^{1}(\Omega) \rightarrow H^{1}(\Omega), \\
\mathcal{V}: H^{-1 / 2}(S) \rightarrow H^{1 / 2}(S)
\end{gathered}
$$

(see [14], Ch. XI, Part B, Sect. 2, Theorem 3 for $\mathcal{V}$ ). This implies that

$$
\mathcal{A}_{0}^{G \mathcal{G}}: H^{1}(\Omega) \times H^{-1 / 2}(S) \rightarrow H^{1}(\Omega) \times H^{1 / 2}(S)
$$

is an invertible operator. Hence, the operator $\mathcal{A}^{G \mathcal{G}}$ possesses the Fredholm property and its index is zero. The injectivity of the operator $\mathcal{A}^{G \mathcal{G}}$, already proved, completes the theorem proof for this operator.

Let us now construct an inverse to operator (14.28). Let $\left(\mathcal{A}^{G \mathcal{G}}\right)^{-1}$ : $H^{1}(\Omega) \times H^{1 / 2}(S) \rightarrow H^{1}(\Omega) \times H^{-1 / 2}(S)$ be the operator inverse to (14.29). 
Thus, for any $\mathcal{F}^{G \mathcal{G}} \in H^{1,0}(\Omega ; \Delta) \times H^{1 / 2}(S)$, the solution of the system $\mathcal{A}^{G \mathcal{G}} \mathcal{U}=\mathcal{F}^{G \mathcal{G}}$ in $H^{1}(\Omega) \times H^{-1 / 2}(S)$ is $\mathcal{U}=\left(\mathcal{A}^{G \mathcal{G}}\right)^{-1} \mathcal{F}^{G \mathcal{G}}$. Taking into account that the operators $V: H^{-1 / 2}(S) \rightarrow H^{1,0}(\Omega ; \Delta)$ and $\mathcal{R}: H^{1}(\Omega) \rightarrow$ $H^{2,0}(\Omega ; \Delta)$ are continuous [9: Th. A.1, Remark B.2], the first equation of this system then implies $u=\mathcal{U}_{1} \in H^{1,0}(\Omega ; \Delta)$ and the operator $\left(\mathcal{A}^{G \mathcal{G}}\right)^{-1}$ is continuous also from $H^{1,0}(\Omega ; \Delta) \times H^{1 / 2}(S)$ to $H^{1,0}(\Omega ; \Delta) \times H^{-1 / 2}(S)$.

The original BVP (14.3)-(14.4) can be written in the form

$$
A^{D} u=F^{D}
$$

where

$$
A^{D}:=\left[\begin{array}{c}
L \\
\tau^{+}
\end{array}\right], \quad F^{D}=\left[\begin{array}{c}
f \\
\varphi_{0}
\end{array}\right] .
$$

The operator $A^{D}: H^{1,0}(\Omega ; L) \rightarrow L_{2}(\Omega) \times H^{1 / 2}(S)$ is evidently continuous and, due to the uniqueness theorem for the BVP, it is also injective.

The invertibility of the operator (14.28) and equivalence Theorem 3 lead to the following assertion.

Corollary. The operator $A^{D}: H^{1,0}(\Omega ; L) \rightarrow L_{2}(\Omega) \times H^{1 / 2}(S)$ is continuous and continuously invertible.

Note that the above statement and the uniqueness Theorem 1 evidently imply the following existence theorem in $H^{1}(\Omega)$.

Theorem 5. Let $\varphi_{0} \in H^{1 / 2}(S)$ and $f \in L_{2}(\Omega)$. Then BVP (14.3)-(14.4) is uniquely solvable in $H^{1}(\Omega)$.

\subsubsection{Integral Equation System $(G \mathcal{T})$}

To obtain a segregated BDIE system of the second kind, we will use equation (14.19) in $\Omega$ and equation (14.21) on $S$, where again the known function $\varphi_{0}$ is substituted for $u^{+}$and an auxiliary unknown function $\psi \in H^{-1 / 2}(S)$ for $T^{+} u$. Then we arrive at the following system $(G \mathcal{T})$ :

$$
\begin{gathered}
u+\mathcal{R} u-V \psi=F_{0} \quad \text { in } \Omega, \\
\frac{1}{2} \psi+T^{+} \mathcal{R} u-\mathcal{W}^{\prime} \psi=T^{+} F_{0} \quad \text { on } S,
\end{gathered}
$$

where $F_{0}$ is given by $(14.25)$.

Let us prove that BVP (14.3)-(14.4) is equivalent to system (14.30)(14.31).

Theorem 6. Let $f \in L_{2}(\Omega)$ and $\varphi_{0} \in H^{1 / 2}(S)$.

(i) If some $u \in H^{1}(\Omega)$ solves $B V P(14.3)-(14.4)$ in $\Omega$, then the couple $(u, \psi)^{\top} \in H^{1}(\Omega) \times H^{-1 / 2}(S)$, where

$$
\psi=T^{+} u \quad \text { on } S,
$$


solves BDIE system (14.30)-(14.31).

(ii) If a couple $(u, \psi)^{\top} \in H^{1}(\Omega) \times H^{-1 / 2}(S)$ solves BDIE system (14.30)(14.31), then the solution is unique, $u$ solves BVP (14.3)-(14.4), and $\psi$ satisfies (14.32).

Proof. As in the proof of Theorem $3, u \in H^{1,0}(\Omega ; \Delta)$ if either hypothesis (i) or (ii) is satisfied.

Let $u \in H^{1}(\Omega)$ be a solution to BVP (14.3)-(14.4). Set $\psi=T^{+} u$. Evidently, $\psi \in H^{-1 / 2}(S)$. Then it immediately follows from relations (14.19) and (14.21) that the couple $(u, \psi)$ solves system (14.30)-(14.31), which completes the proof of item (i).

Let now a couple $(u, \psi) \in H^{1}(\Omega) \times H^{-1 / 2}(S)$ solve BDIE system (14.30)(14.31).

Take the conormal derivative of equation (14.30) on $S$ and subtract it from equation (14.31) to obtain

$$
\Psi^{*}:=\psi-T^{+} u=0 \quad \text { on } S,
$$

that is, equation (14.32) is proved.

Equation (14.30) and Lemma 1 with $\Psi=\psi$ and $\Phi=\varphi_{0}$ imply that $u$ is a solution of equation (14.1) and

$$
V \Psi^{*}(y)-W \Phi^{*}(y)=0, \quad y \in \Omega,
$$

where $\Phi^{*}=\varphi_{0}-u^{+}$. Since $\Psi^{*}=0$ on $S$, (14.33) reduces to

$$
W \Phi^{*}(y)=0, \quad y \in \Omega,
$$

and Lemma 2(ii) implies that $\Phi^{*}=0$ on $S$. This means that $u$ satisfies the Dirichlet condition (14.4).

By Remark 1, the unique solvability of BDIE system (14.30)-(14.31) then follows from (14.32) along with the unique solvability of BVP (14.3)-(14.4).

System (14.30)-(14.31)) can be rewritten in the form

$$
\mathcal{A}^{G \mathcal{T}} \mathcal{U}=\mathcal{F}^{G \mathcal{T}}
$$

where $\mathcal{U}^{\top}:=(u, \psi)^{\top} \in H^{1}(\Omega) \times H^{-1 / 2}(S)$,

$$
\mathcal{A}^{G \mathcal{T}}:=\left[\begin{array}{cc}
I+\mathcal{R} & -V \\
T^{+} \mathcal{R} & \frac{1}{2} I-\mathcal{W}^{\prime}
\end{array}\right], \quad \mathcal{F}^{G \mathcal{T}}:=\left[\begin{array}{c}
F_{0} \\
T^{+} F_{0}
\end{array}\right] .
$$

Due to the mapping properties of the operators involved in (14.34) we have $\mathcal{F}^{G \mathcal{T}} \in H^{s, 0}\left(\Omega^{+} ; \Delta\right) \times H^{-1 / 2}(S)$, and the operators

$$
\begin{aligned}
\mathcal{A}^{G \mathcal{T}} & : H^{1,0}(\Omega ; \Delta) \times H^{-1 / 2}(S) \rightarrow H^{1,0}(\Omega ; \Delta) \times H^{-1 / 2}(S) \\
& : H^{1}(\Omega) \times H^{-1 / 2}(S) \rightarrow H^{1}(\Omega) \times H^{-1 / 2}(S)
\end{aligned}
$$

are continuous. By Theorem 6 and the uniqueness Theorem 1, both operators (14.35) and (14.36) are injective. 
Theorem 7. Operators (14.35) and (14.36) are continuous and invertible.

Proof. The operator

$$
\mathcal{A}_{0}^{G \mathcal{T}}:=\left[\begin{array}{cc}
I & -V \\
0 & \frac{1}{2} I
\end{array}\right]
$$

is a compact perturbation of both operators (14.35) and (14.36), due to compactness properties of the operators $\mathcal{R}$ and $\mathcal{W}$ (see [7], [8], and [10]). The invertibility of operators (14.35) and (14.36) then follows by arguments similar to those in the proof of Theorem 4.

\subsection{United Boundary-domain Integro-differential Equations and Problems}

Instead of introducing an auxiliary function $\psi$, we will work in this section with the original form of the equations containing the conormal derivative operator $T^{+}$of the internal field.

\subsubsection{Integro-differential Problem $(G D)$}

The equivalence of the differential and boundary-domain integro-differential equations proved in Theorem 2 allows us to supplement BDIDE (14.19) with the original Dirichlet boundary conditions and arrive at BDIDP $(G D)$ constituted by (14.19), (14.4). The BDIDP is equivalent to the Dirichlet boundary value problem (14.3)-(14.4) in $\Omega$, in the following sense.

Theorem 8. Let $f \in L_{2}(\Omega), \varphi_{0} \in H^{1 / 2}(S)$. A function $u \in H^{1,0}(\Omega ; \Delta)$ solves BVP (14.3)-(14.4) in $\Omega$ if and only if u solves BDIDP (14.19), (14.4). Such a solution does exist and is unique.

Proof. A solution of BVP (14.3)-(14.4) does exist and is unique, by Theorem 5, and provides a solution to BDIDP (14.19), (14.4), by Theorem 2. On the other hand, any solution of BDIDP (14.19), (14.4) also satisfies (14.3), due to the same Theorem 2.

BDIDP (14.19), (14.4) can be written in the form

$$
\mathcal{A}^{G D} u=\mathcal{F}^{G D}
$$

where

$$
\mathcal{A}^{G D}:=\left[\begin{array}{c}
I+\mathcal{R}-V T^{+}+W \tau^{+} \\
\tau^{+}
\end{array}\right], \quad \mathcal{F}^{G D}=\left[\begin{array}{c}
\mathcal{P} f \\
\varphi_{0}
\end{array}\right] .
$$

Owing to the mapping properties of operators $V, W, \mathcal{P}$, and $\mathcal{R}$, we have $\mathcal{F}^{G D} \in H^{1,0}(\Omega ; \Delta) \times H^{1 / 2}(S)$, and the operator $\mathcal{A}^{G D}: H^{1,0}(\Omega ; \Delta) \rightarrow$ $H^{1,0}(\Omega ; \Delta) \times H^{1 / 2}(S)$ is continuous. By Theorem 8 , it is also injective. Let us now characterize the range of the operator $\mathcal{A}^{G D}$ in the whole space $H^{1,0}(\Omega ; \Delta) \times H^{1 / 2}(S)$. 
Theorem 9. Let

$$
\mathcal{F}^{G D}=\left(\mathcal{F}_{1}^{G D}, \mathcal{F}_{2}^{G D}\right) \in H^{1,0}(\Omega ; \Delta) \times H^{1 / 2}(S) .
$$

System (14.37) has a solution in $H^{1,0}(\Omega ; \Delta)$ if and only if there exists $f_{*} \in L_{2}(\Omega)$ such that

$$
\mathcal{F}_{1}^{G D}=\mathcal{P} f_{*} \quad \text { in } \Omega .
$$

When the solution does exist, it is unique.

Proof. If condition (14.38) is satisfied, then, according to Theorem 7, there exists a unique solution of system (14.37).

On the other hand, if $u \in H^{1,0}(\Omega ; \Delta)$ is a solution of system (14.37), then it satisfies the third Green identity (14.18). Comparing it with the first equation of system (14.37) implies representation (14.38) with $f_{*}=L u$.

Let $T_{\Delta}^{+}, V_{\Delta}$, and $W_{\Delta}$ denote the operators of the conormal derivative, single-layer potential and double-layer potential associated with the Laplace operator, that is, for the coefficient $a=1$.

Remark 2. Condition (14.38) for an $\mathcal{F}_{1}^{G D} \in H^{1,0}(\Omega ; \Delta)$ is equivalent to the condition

$$
V_{\Delta} T_{\Delta}^{+}\left(a \mathcal{F}_{1}^{G D}\right)-W_{\Delta}\left(a \mathcal{F}_{1}^{G D}\right)^{+}=0 \quad \text { in } \Omega
$$

or

$$
V\left[T^{+} \mathcal{F}_{1}^{G D}+\mathcal{F}_{1}^{G D+} \frac{\partial a}{\partial n^{+}}\right]-W\left(\mathcal{F}_{1}^{G D}\right)^{+}=0 \quad \text { in } \Omega .
$$

Indeed, condition (14.38) can be rewritten as

$$
a \mathcal{F}_{1}^{G D}=\mathcal{P}_{\Delta} f_{*} \quad \text { in } \Omega .
$$

The third Green identity (14.18) for $u=a \mathcal{F}_{1}^{G D}$ and for the potentials associated with the operator $\Delta$ gives

$$
a \mathcal{F}_{1}^{G D}-V_{\Delta} T_{\Delta}^{+}\left(a \mathcal{F}_{1}^{G D}\right)+W\left(a \mathcal{F}_{1}^{G D}\right)^{+}=\mathcal{P}_{\Delta} \Delta\left(a \mathcal{F}_{1}^{G D}\right) \text { in } \Omega .
$$

Thus (14.39) implies (14.41) with $f_{*}=\Delta\left(a \mathcal{F}_{1}^{G D}\right)$.

On the other hand, if (14.41) is satisfied, then application of the Laplace operator to it gives $\Delta\left(a \mathcal{F}_{1}^{G D}\right)=f_{*}$. Substituting this into (14.42) and comparing with (14.41) implies (14.39).

Condition (14.40) follows from (14.39) and the definitions of $V$ and $W$.

To realize how restrictive condition (14.38), or, equivalently, conditions (14.39) and (14.40), are, we prove the following assertion. 
Lemma 3. For any function $\mathcal{F}_{1} \in H^{1,0}(\Omega ; \Delta)$, there exists a unique couple $\left(f_{*}, \Phi_{*}\right)=\mathcal{C}_{\Phi} \mathcal{F}_{1} \in L_{2}(\Omega) \times H^{1 / 2}(S)$ such that

$$
\mathcal{F}_{1}(y)=\mathcal{P} f_{*}(y)-W \Phi_{*}(y), \quad y \in \Omega,
$$

and $\mathcal{C}_{\Phi}: H^{1,0}(\Omega ; \Delta) \rightarrow L_{2}(\Omega) \times H^{1 / 2}(S)$ is a linear bounded operator.

Proof. We adapt here the proof scheme from [8], Lemma 5.2.

Suppose first that there exist some functions $f_{*}(y), \Phi_{*}(y)$ satisfying (14.43) and find their expressions in terms of $\mathcal{F}_{1}(y)$. Taking into account definitions (14.7) and (14.9) for the volume and double layer potentials, ansatz (14.43) can be rewritten as

$$
a(y) \mathcal{F}_{1}(y)=\mathcal{P}_{\Delta} f_{*}(y)-W_{\Delta}\left[a \Phi_{*}\right](y), \quad y \in \Omega,
$$

where $\mathcal{P}_{\Delta}=\left.\mathcal{P}\right|_{a=1}, W_{\Delta}=\left.W\right|_{a=1}$ are the volume and double layer potential operators associated with the Laplace operator $\Delta$.

Applying the Laplace operator to (14.44) we obtain that

$$
f_{*}=\Delta\left(a \mathcal{F}_{1}\right) \text { in } \Omega \text {. }
$$

Then (14.44) can be rewritten as

$$
W_{\Delta}\left[a \Phi_{*}\right](y)=Q(y), \quad y \in \Omega,
$$

where

$$
Q(y):=\mathcal{P}_{\Delta}\left[\Delta\left(a \mathcal{F}_{1}\right)\right](y)-a(y) \mathcal{F}_{1}(y), \quad y \in \Omega .
$$

The trace of (14.46) on the boundary gives

$$
\left[-\frac{1}{2} I+\mathcal{W}_{\Delta}\right]\left[a \Phi_{*}\right](y)=Q^{+}(y), \quad y \in S,
$$

where $\mathcal{W}_{\Delta}:=\left.\mathcal{W}\right|_{a=1}$ is the direct value on $S$ of the double layer operator associated with the Laplace operator.

Since $\left[-(1 / 2) I+\mathcal{W}_{\Delta}\right]$ is an isomorphism (see, e.g., [14], Ch. XI, Part B, Sect. 2, Remark 8$)$ and $a(y) \neq 0$, we obtain the following expression for $\Phi_{*}$ :

$$
\Phi_{*}(y)=\frac{1}{a(y)}\left[-\frac{1}{2} I+\mathcal{W}_{\Delta}\right]^{-1} Q^{+}(y), \quad y \in S,
$$

Now we have to prove that $f_{*}(y), \Phi_{*}(y)$ given by (14.45) and (14.49) do satisfy (14.43). Indeed, the potential $W_{\Delta}\left[a \Phi_{*}\right](y)$ with $\Phi_{*}(y)$ given by (14.49) is a harmonic function, and one can check that $Q$ given by (14.47) is also harmonic. Since (14.48) implies that they coincide on the boundary, the two harmonic functions should also coincide in the domain, i.e., (14.46) holds true, which implies (14.43).

Thus we constructed a bounded operator $\mathcal{C}_{\Phi}: H^{1,0}(\Omega ; \Delta) \rightarrow L_{2}(\Omega) \times$ $H^{1 / 2}(S)$ given by (14.45), (14.49), (14.47).

Lemma 3 implies that ansatz (14.38) does not cover the whole space $H^{1,0}(\Omega ; \Delta)$. 


\subsubsection{Integro-differential Equation $(G)$}

In this section, we eliminate the Dirichlet boundary condition to deal with only one integro-differential equation. Substituting the Dirichlet boundary condition (14.4) into (14.19) leads to the following $\operatorname{BDIE}(G)$ for $u \in$ $H^{1,0}(\Omega ; \Delta)$ :

$$
\mathcal{A}^{G} u:=u+\mathcal{R} u-V T^{+} u=\mathcal{F}^{G} \quad \text { in } \Omega,
$$

where

$$
\mathcal{F}^{G}=F_{0}=\mathcal{P} f-W \varphi_{0} .
$$

Let us prove the equivalence of the BDIDE to the BVP (14.3)-(14.4).

Theorem 10. Let $f \in L_{2}(\Omega), \varphi_{0} \in H^{1 / 2}(S)$. A function $u \in H^{1,0}(\Omega ; \Delta)$ solves the mixed BVP (14.3)-(14.4) in $\Omega$ if and only if $u$ solves BDIDE (14.50) with right-hand side (14.51). Such a solution exists and is unique.

Proof. Any solution of BVP (14.3)-(14.4) solves BDIDE (14.50) due to the third Green formula (14.19).

On the other hand, if $u$ is a solution of BDIDE (14.50), then Lemma 1 implies that $u$ satisfies equation (14.3) and $W\left(\varphi_{0}-u^{+}\right)=0$ in $\Omega$. Lemma 2(ii) then implies that the Dirichlet boundary condition (14.4) is satisfied. Thus any solution of BDIDE (14.50) satisfies BVP (14.3)-(14.4). The unique solvability of the latter is implied by Theorem 5 .

The mapping properties of operators $V, W, \mathcal{P}$, and $\mathcal{R}$ imply the membership $\mathcal{F}^{G} \in H^{1,0}(\Omega ; \Delta)$ and continuity of the operator $\mathcal{A}^{G}$ in $H^{1,0}(\Omega ; \Delta)$, while Theorem 10 implies its injectivity.

Theorem 11. The operator $\mathcal{A}^{G}$ is continuous and continuously invertible in $H^{1,0}(\Omega ; \Delta)$.

Proof. The continuity of $\mathcal{A}^{G}$ is already proved, and we have to prove the existence of a bounded inverse operator $\left(\mathcal{A}^{G}\right)^{-1}$.

Let us consider equation (14.50) with an arbitrary function $\mathcal{F}^{G}$ from $H^{1,0}(\Omega ; \Delta)$. By Lemma $3, \mathcal{F}^{G}$ can be presented as

$$
\mathcal{F}^{G}(y)=\mathcal{P} f_{*}(y)-W \Phi_{*}(y) \quad y \in \Omega
$$

where $\left(f_{*}, \Phi_{*}\right)=\mathcal{C}_{\Phi} \mathcal{F}^{G}$ and $\mathcal{C}_{\Phi}$ is a bounded operator from $H^{1,0}(\Omega ; \Delta)$ to $L_{2}(\Omega) \times H^{1 / 2}(S)$. Then Theorem 10 and the Corollary imply that equation (14.50) has a unique solution $u=\left(A^{D}\right)^{-1}\left(f_{*}, \Phi_{*}\right)^{\top}$, where $\left(A^{D}\right)^{-1}$ is a bounded operator.

\subsection{Concluding Remarks}

The Dirichlet problem for a variable-coefficient PDE with a right-hand side function from $L_{2}(\Omega)$, and with the Dirichlet data from the spaces $H^{1 / 2}(S)$, has been considered in the paper. It was shown that the BVP can be equivalently reduced to two direct segregated boundary-domain integral equation systems, one of them of the second kind. On the other hand, the 
BVP can be equivalently reduced to a united boundary-domain integrodifferential problem, or to a united boundary-domain integro-differential equation of the second kind. It was shown that the operators associated with the left-hand sides of all the four systems/problems/equations are continuous and three of them continuously invertible in the corresponding Sobolev-Slobodetski spaces.

A further analysis of spectral properties of the two second kind equations obtained in the paper is needed to decide whether the resolvent theory and the Neumann series method (see [16], [17], and references therein) are efficient for solving the equations.

By the same approach, the corresponding BDIDEs/BDIDPs for unbounded domains can be analyzed as well. The approach can also be extended to more general PDEs and to systems of PDEs, while smoothness of the variable coefficients and the boundary can be essentially relaxed.

This study can serve as a starting point for approaching BDIDEs/ BDIDPs based on the localized parametrices, leading after discretization to sparsely populated systems of linear algebraic equations, attractive for computations [9]. This can then be extended to analysis of localized BDIDEs/BDIDPs of nonlinear problems [18].

\section{References}

1. P.A. Martin, J.D. Richardson, L.J. Gray, and J.R. Berger, On Green's function for a three-dimensional exponentially graded elastic solid, Proc. Roy. Soc. London A458 (2002), 1931-1947.

2. E.E. Levi, I problemi dei valori al contorno per le equazioni lineari totalmente ellittiche alle derivate parziali, Mem. Soc. Ital. dei Sci. XL 16 (1909), 1-112.

3. D. Hilbert, Grundzüge einer allgemeinen Theorie der linearen Integralgleichungen, Teubner, Leipzig, 1912.

4. C. Miranda, Partial Differential Equations of Elliptic Type, 2nd ed., Springer-Verlag, Berlin-Heidelberg-New York, 1970.

5. A. Pomp, Levi functions for linear elliptic systems with variable coefficients including shell equations, Computational Mech. 22 (1998), 93-99.

6. A. Pomp, The Boundary-domain Integral Method for Elliptic Systems with Applications in Shells, Lect. Notes in Math. 1683, Springer-Verlag, Berlin-Heidelberg, 1998.

7. O. Chkadua, S.E. Mikhailov, and D. Natroshvili, Analysis of direct boundary-domain integral equations for a mixed BVP with variable coefficient. I (submitted for publication), preprint available from http:// www.gcal.ac.uk/cms/contact/staff/sergey/CMS-MAT-PP-2004-1.pdf.

8. O. Chkadua, S.E. Mikhailov, and D. Natroshvili, Analysis of direct boundary-domain integral equations for a mixed BVP with variable coefficient. II (submitted for publication), preprint available from http:// www.gcal.ac.uk/cms/contact/staff/sergey/CMS-MAT-2004-12.pdf. 
9. S.E. Mikhailov, Localized boundary-domain integral formulation for problems with variable coefficients, Engng. Analysis Boundary Elements 26 (2002), 681-690.

10. S.E. Mikhailov, Analysis of united boundary-domain integro-differential and integral equations for a mixed BVP with variable coefficient (submitted for publication), preprint available from http://www.gcal. ac.uk/ cms/contact/staff/sergey/CMS-MAT-2004-11.pdf.

11. J.-L. Lions and E. Magenes, Non-homogeneous Boundary Value Problems and Applications, vol. 1, Springer-Verlag, Berlin-Heidelberg-New York, 1972.

12. H. Triebel, Interpolation Theory, Function Spaces, Differential Operators, North-Holland, Amsterdam, 1978.

13. M. Costabel, Boundary integral operators on Lipschitz domains: elementary results, SIAM J. Math. Anal. 19 (1988), 613-626.

14. R. Dautray and J.-L. Lions, Mathematical Analysis and Numerical Methods for Science and Technology, vol. 4: Integral Equations and Numerical Methods, Springer-Verlag, Berlin-Heidelberg, 1990.

15. W. McLean, Strongly Elliptic Systems and Boundary Integral Equations, Cambridge Univ. Press, Cambridge, 2000.

16. S.E. Mikhailov, On an integral equation of some boundary value problems for harmonic functions in plane multiply connected domains with nonregular boundary, Mat. Sb. 121 (1983), 533-544. (English translation: USSR Math.-Sb. 49 (1984), 525-536.)

17. O. Steinbach and W.L. Wendland, On C. Neumann's method for second-order elliptic systems in domains with non-smooth boundaries, J. Math. Anal. Appl. 262 (2001), 733-748.

18. S.E. Mikhailov, Localized direct boundary-domain integro-differential formulations for scalar nonlinear problems with variable coefficients, J. Engng. Math. 51 (2005), 283-302. 